\title{
Visualization of the Short Term Maximum Lyapunov Exponent Topography in the Epileptic Brain
}

\author{
Nadia Mammone, Student Member, IEEE, Francesco Carlo Morabito, Senior Member, IEEE, \\ and José C. Principe, Fellow, IEEE
}

\begin{abstract}
In this paper, a new kind of brain topography is introduced and applied to data from four patients affected by intractable epilepsy. Experience has shown that the short term maximum Lyapunov exponent (STLmax) is a robust parameter when optimized for the dynamical analysis of the Electroencephalography (EEG). The objective of this work is to map the spatial distribution of STLmax over time. STLmax is estimated from segments of each channel of long term continuous scalp EEG recordings and a movie of the STLmax segment estimates is created over the head. Movies allow for a simple visualization of which electrodes are related to the highest or lowest chaoticity for the longest time. We found out that the interictal epileptiform activity is related to the highest STLmax level, whereas the focal area is related to low STLmax levels during either the interictal and preictal stages.
\end{abstract}

\section{INTRODUCTION}

$\mathrm{E}^{\mathrm{s}}$ lectroencephalographic (EEG) brain topography was born at the end of the $80 \mathrm{~s}$. It consists of plotting the EEG in 2-D maps by color coding EEG features, most commonly the EEG power. This approach gives a helpful and representative view of alterations of the frequency and amplitude of EEG co registered to the scalp location. Topographic analysis helped clinicians to reach diagnostics [1-3], because they can easily pinpoint the exact location of EEG alterations. In particular, EEG topography has been widely used as a tool for investigating the activity of epileptic brains [4-12]. The EEG recorded from an epileptic brain can be classified in several stages: the data segment which starts with the seizure onset and ends with the seizure, is labeled as "ictal". The "preictal" stage is the data segment preceding the seizure onset, whereas the "postictal" stage is the period following the end of seizure. The period between the postictal stage of one seizure and the preictal stage of the next seizure is called "interictal" stage.

As proved by the results reported by different research groups working on epileptic seizure prediction, the epileptic seizure is not a completely random and unpredictable event. Iasemidis et al. [13] achieved a sensitivity of $84 \%$ with a false prediction rate of 0.12 /hour with intracranial EEG data

N.Mammone and F.C.Morabito are with Department of Informatics, Mathematics, Electronics and Transportations (DIMET), University of Reggio Calabria, ITALY (phone: +39-0965-875285; fax: +39-0965875220; e-mail: nadia.mammone@ing.unirc.it, morabito@unirc.it).

J.C.Principe is with Departments of Electrical and Computer Engineering and Biomedical Engineering, University of Florida, Gainesville, FL 32611-6200 USA (e-mail: principe@cnel.ufl.edu). from patients affected by temporal lobe epilepsy. Since it seems possible to anticipate reliably seizure occurrence, a gradual transformation of brain activity leading to a seizure can be hypothesized: a seizure occurs when a massive group of neurons in the cerebral cortex begins to discharge in a highly organized rhythmic pattern, then it develops according to some poorly described dynamics. Thus, it is reasonable to wonder whether a long term analysis of brain activity over the cortex might provide information about this evolution, as well as about the epileptogenic focus. The parameter we chose for the mapping was the Short-Term maximum Lyapunov exponent (STLmax). Iasemidis et al. demonstrated that the intracranial EEG signals recorded from patients with refractory temporal lobe epilepsy have properties resembling the characteristic of non convergent dynamical systems since: (1) they are non-linear [15]; (2) they have a non-integer (fractal) dimension [16-17]; (3) there is at least one positive Lyapunov exponent [18-19]. These investigators found the STLmax to be particularly useful for evaluating the dynamical behavior of the EEG signal over time. STLmax is noted as "short-term" because it is calculated iteratively over $10.24 \mathrm{sec}$ non-overlapping windows. The algorithm assumes stationarity but discards fast activity within the epoch (for example, short-term bursts, like interictal spikes, do not bias the estimation of the STLmax [15]). STLmax is calculated per electrode and it is a measure of how orderly the underlying system (i.e. the brain) is. In particular, STLmax usually starts dropping even before the preictal stage, continuing to drop until the system enters the ictal stage, and it starts increasing again in the postictal stage. Because of its features, we chose STLmax as the parameter for the topographic maps. However, very little is known about the spatial distribution over time of the STLmax, therefore, this study will elucidate the long term distribution of the STLmax over the scalp.

The paper is organized as follows: Section II presents the data and the methods, Section III presents the results and Section IV reports the conclusions. 
TABLE I

THE NUMBERS ASSOCIATED TO THE LABELS OF THE ELECTRODES FOR SCALP EEG

\begin{tabular}{cccccccccccc}
\hline \hline label & MN1 & MN2 & FP1 & FP2 & F7 & F8 & F3 & F4 & T7 & T8 & C3 \\
number & 1 & 2 & 3 & 4 & 5 & 6 & 7 & 8 & 9 & 10 & 11 \\
label & C4 & P7 & P8 & P3 & $\mathbf{P 4}$ & O1 & O2 & Fz & Cz & A1 & A2 \\
number & 12 & 13 & 14 & 15 & 16 & 17 & 18 & 19 & 20 & 21 & 22 \\
\hline \hline
\end{tabular}

TABLE II

THE NUMBERS ASSOCIATED TO THE LABELS OF THE ELECTRODES FOR IEEG

\begin{tabular}{|c|c|c|c|c|c|c|c|c|c|c|c|c|c|c|}
\hline label & LD1 & $\begin{array}{l}\text { LD3 } \\
\end{array}$ & LD5 & LD7 & $\begin{array}{l}\text { LD9 } \\
\end{array}$ & LD11 & RD2 & RD4 & RD6 & RD8 & RD10 & RD12 & $\begin{array}{ll}\text { LT1 } \\
\end{array}$ & $\overline{~ L T 2 ~}$ \\
\hline number & 1 & 2 & 3 & 4 & 5 & 6 & 7 & 8 & 9 & 10 & 11 & 12 & 13 & 14 \\
\hline label & LT3 & LT4 & RT1 & RT2 & RT3 & RT4 & LF1 & LF2 & LF3 & LF4 & RF1 & RF2 & RF3 & RF4 \\
\hline number & 15 & 16 & 17 & 18 & 19 & 20 & 21 & 22 & 23 & 24 & 25 & 26 & 27 & 28 \\
\hline
\end{tabular}

\section{Methodology}

\section{A. The data}

The data are from four patients affected by intractable epilepsy: two patients (patients A and B) were monitored through multi-channel EEG scalp electrodes whereas the other two (patients $\mathrm{C}$ and D) were monitored through intracranial electrodes. The data were acquired by means of a Nicolet BMSI 4000 recording system, the amplifiers were set in the input range of $\pm 0.6 \mathrm{mV}$ and the frequency range of the filters was set at $0.5-70 \mathrm{~Hz}$. The signals were sampled at $200 \mathrm{~Hz}$, using an analog to digital converter with 10 -bit quantization, and they were finally stored. The scalp recordings are from 23 channels utilizing hemispheric derivations, according to the 10-20 system whereas the intracranial recordings are from 28 electrodes implanted in the hippocampus, temporal and frontal lobe cortexes (see Tables 1 and 2). The interictal segments were extracted by seizure detection programs with the technologist's screening and the physician's review. The dataset we used for the mapping consist, for each patient, in a 60 min data segment prior the onset of a seizure plus a $5 \mathrm{~min}$ segment including the seizure and the post-ictal stage.

\section{B. The Short Term Maximum Lyapunov Exponent}

In order to estimate the STLmax, a modified Wolf algorithm is implemented here. In particular, the embedding dimension $p$ was set at 7 and the time lag $\tau$ was set at $20 \mathrm{msec}$ according to [20]. The details of the STLmax algorithm are described in [21-23].

\section{STLmax topographic maps}

Because of its features, the STLmax profile is the choice of the topography map. For each channel, we estimated a STLmax profile where one sample corresponds to $10.24 \mathrm{sec}$. This new time series was smoothed by overlapping six STLmax samples, in other words smoothing through $1 \mathrm{~min}$ overlapping time windows. We arranged the smoothed STLmax time series as rows of a matrix whose $n \times 1$ column represents the set of the STLmax values associated with the $n$ electrodes at a certain time point $t$. We aimed to compare the STLmax topographic map with the topography of the EEG power of the same electrodes. Thus we also estimated the power of each EEG channel downsampled to the
STLmax time resolution within 1min moving windows, in order to get a smooth and comparative profile. The EEG power time series were also arranged as rows of a matrix. For every patient we selected one hour segment before the onset of each seizure including the ictal and post ictal periods, and both the STLmax and the EEG power were estimated. For each time window (i.e. column of the matrices), we plotted a 2-D map for the EEG power and a 2D map for STLmax, exploiting a function for 2-D plot from the toolbox EEGLAB [14] and adapting the electrode location according to the montage used for the patients. For each electrode, the corresponding value was plotted, encoding the values according to a continuous color scale going from blue (low values) to red (high values). The range of the scale was fixed, so that we could detect the overall variation over time as well as the local in time STLmax spatial distribution. The color of the spatial points lying between the electrodes are calculated by interpolation (calculating intermediary values on the basis on the value of its neighbors), and thus a smooth gradation of colors is achieved. All the figures were captured as frames of a movie and each frame was associated to the corresponding time, so that it was possible to keep the time information and therefore to identify the critical stages while reviewing the movie.

\section{Mapping review and quantification}

Reviewing the movies, we analyzed whether the STLmax mapping was able to provide information about the abnormal epileptiform activity during the interictal and ictal stages. During the visualization we followed the topographic trends of the most active areas in terms of lowest and highest STLmax levels, in other words, we looked for the electrodes that had been associated with low-STLmax or high-STLmax values for the longest time. and we called these electrodes the "most active". We clustered the electrodes in a lowactivity, a high-activity and a neutral cluster, according to both EEG power and STLmax. We included the four most low-active electrodes in the first cluster, the four most highactive electrodes in the second cluster and the rest in the neutral cluster. In order to quantify the visual review of the movies, we estimated the time each electrode was most active in terms of either EEG power and STLmax finding out how many times the electrodes belonged to the low- and high-activity clusters. In our $60 \mathrm{~min}$ dataset there are 352 
overlapping windows (the width of the window is $10.24 \mathrm{sec}$ ) and the time estimation was quantified in terms of number of temporal windows.

TABLE III

SUMMARY OF THE QUANTIFICATION

\begin{tabular}{cllll}
\hline \hline Patient & $\begin{array}{l}\text { low-EEG } \\
\text { power } \\
\text { electrodes }\end{array}$ & $\begin{array}{l}\text { low-STLmax } \\
\text { electrodes }\end{array}$ & $\begin{array}{l}\text { high-EEG } \\
\text { power } \\
\text { electrodes }\end{array}$ & $\begin{array}{l}\text { high- } \\
\text { STLmax } \\
\text { electrodes }\end{array}$ \\
A & P3, Cz, A1 & P7, Cz, A1 & $\begin{array}{l}\text { F3, C3, O2, } \\
\text { FP1, FP2, F7, }\end{array}$ & $\begin{array}{l}\text { Fz } \\
\text { B }\end{array}$ \\
& Cz, A1, P3, & MN1, P7, P8, & F3, C3, O2, & FP1, FP2, F7, \\
& P4 & Cz & Fz & F8 \\
C & LD3, LT1, & LD7, LD9, & LD9, LD11, & RD6, RD10, \\
& RT1, LF3 & RD2, RT2, & RD12, RF1 & LF2, LF3, \\
& & RT3, RT4 & & LF4, RF1 \\
D & RD12, LT2, & RD2, RD4, & LD1, LD7, & LD1, LD3, \\
& RT2, RT3, & LT2 & LF1 & RD10, RT1, \\
& RT4, LF2, & & & \\
& LF3, RF2, & & & \\
\hline \hline
\end{tabular}

\section{RESULTS}

This section presents the results of the monitoring and quantification of the STLmax mapping compared to the EEG power mapping. The movies are available at: www.permano.it/movies/home.php, whereas Figure 1 reports the quantification of the time each electrode belonged to the low- and high-activity clusters. Table III summarizes the results of the quantification. We will discuss the results patient by patient. Patient A: The electrographical review of the interictal segments reports active epileptiform potentials consisting of moderate voltage spike and wave complexes were recorded independently from the right front-temporal region as well as the left fronttemporal region with a ratio of right to left of 50:50. Every event appeared bilaterally from the anterior temporal region (mainly from T7 but also from F7) with higher amplitude on the left, except the first event (seizure induced by hyperventilation) that had a balanced onset and that was non-lateralizing, all the events localized on the left anterior mesial temporal lobe. We found out that the left temporal region (P7, A1) and Cz were the most active low-STLmax areas, even though they were not stable over time, whereas the most active high-STLmax area was stable and concentrated in the fronto-temporal region (FP1, FP2, F7, F8). Low-EEG power was most of the time located in the left tempo-parietal region $(\mathrm{P} 3, \mathrm{~A} 1, \mathrm{Cz})$ whereas the highEEG power moved within the central area. Thus highSTLmax distribution was concentrated in the area of the inter-ictal activity and appeared very stable during the movie review, while the low STLmax was located over the focal area. Low-EEG power moved near the region of the onset too (P3 and A1), but there was not any peculiar correlation for the inter-ictal activity in the EEG power mapping. Thus STLmax appears more sensitive to either the inter-ictal and the ictal epileptiform activity.

Patient B: Every event rose bilaterally, as well as interictal spikes and waves, and was non-localizing. We found out that the right and left temporal regions (P7, P8) and $\mathrm{Cz}$ were the most active low-STLmax area and, once again, the most active high-STLmax area was stable and concentrated in the front-temporal region (FP1, FP2, F7, F8). Low-EEG power was mainly located in the left tempo-parietal region (P3, P4, A1, Cz) whereas the high-EEG power moved within the central area. STLmax shows evidence of bilateral activity, in particular due to the distribution of the high levels but EEG power provides no evidence of bilateral activity. Patient C: Electrographically, the interictal segments of patient $\mathrm{C}$ are characterized by active spikes and wave activities coming from the right and left orbital frontal regions. All the events came from the right hippocampus, in particular from RD2, the right temporal leads were always soon involved. Looking at the quantification of the distribution we can verify that the RD2 and RT leads, together with LD7 and LD9, were most of the time associated with low-STLmax. The LD leads are worth being inspected electrographically in order to detect any relationship with the onset. LF and RF leads, together with RD6 and RD10, had high-STLmax activity. Therefore the focus appears associated with low-STLmax and the interictal activity with high-STLmax. The focus has no peculiar relation when seen with the EEG power mapping. Reviewing the movies for this patient, we detected a pretty stable STLmax distribution. Patient D: For this patient, interictal spikes and waves came from right temporal depths, right temporal leads and intermittently from orbital-frontal. Every event came from right mesial temporal lobe (RD2 and RD4). Reviewing the movies of patient $\mathrm{D}$, we detected a very stable STLmax distribution. Looking at Figure 1, we can see that the high-STLmax area was concentrated in RD10, RT1, RF3 (in agreement with the location of interictal activity) and LD1, LD3. The onset area (RD2 and RD4) was permanently related to low-STLmax levels. The EEG power movie shows a pretty stable distribution, but the onset area and the interictal activity are not associated to any peculiar color map, as shown by the quantification in Figure 1.

\section{CONCLUSIONS}

In this paper, the STLmax was mapped for a long term monitoring of the brain of four patients affected by intractable epilepsy. We created movies from the mapping and the movies allowed us to follow the dynamics of the brain over time, in particular, quantifying the information we got from visual inspection, we found out that the onset areas are often associated with low-STLmax activity, whereas the areas associated with interictal epileptiform activity are associated with high-STLmax activity. The topographic map constructed with EEG power shows much weaker spatial relationships with the epileptic activity. The STLmax mapping might provide important information about the dynamics of the epileptic brain and about the evolution of the epileptic seizures, following this evolution from the interictal epileptiform activity until the onset of the epileptic event, but further work is necessary. 

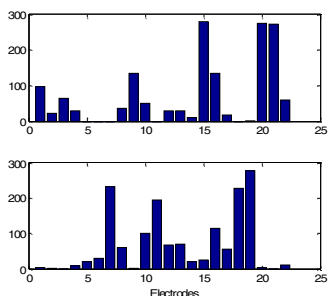

(a.1)
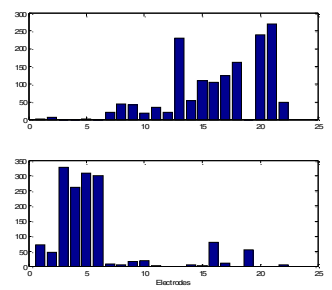

(a.2)
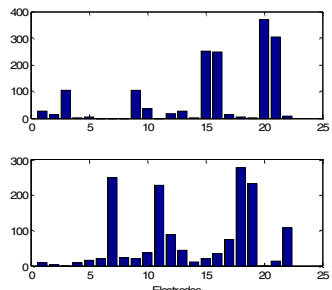

(b.1)
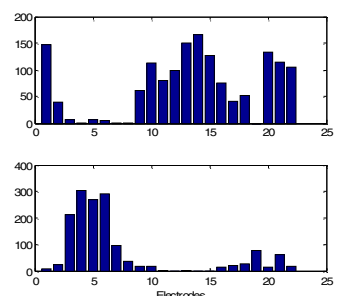

(b.2)
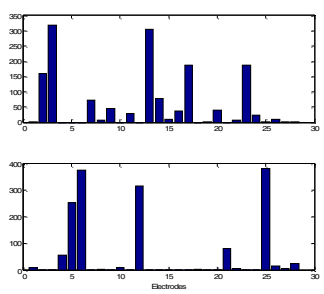

(c.1)
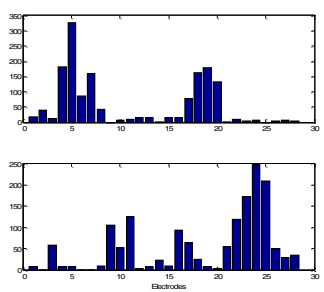

(c.2)
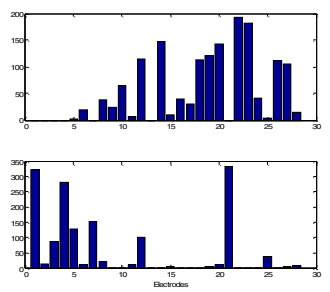

(d.1)
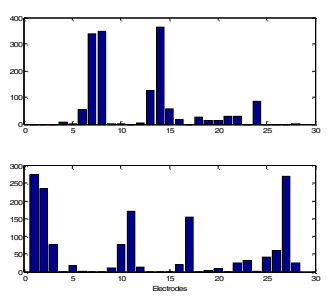

(d.2)

Fig. 1. Figures x.1: Quantification of the time each electrode $x$ belonged to the low-activity (top) and high-activity (bottom) clusters according to the EEG power mapping. Figures x.2: Quantification of the time each electrode $x$ belonged to the low-activity (top) and high-activity (bottom) clusters according to STLmax mapping.

\section{ACKNOWLEDGEMENT}

This work was partially supported by NIH grant on Brain Dynamics, NIH R01 NS 39687.

\section{REFERENCES}

[1] R.N. Harner, "Clinical application of computed EEG topography", Topographic Mapping of Brain Electrical Activity (Ed. F.H. Duffy) Boston, Butterworths, 1986, pp. 347-356.

[2] M.R. Nuwer, "Quantitative EEG", Journal of Clinical Neurophysiology, 1988; 5: 1-86.

[3] C. Babiloni, G. Binetti, E. Cassetta, D. Cerboneschi, G. Dal Forno, C. Del Percio, F. Ferreri, R. Ferri, B. Lanuzza, C. Miniussi, D. V. Moretti, F. Nobili, R. D. Pascual-Marqui, G. Rodriguez, G. Romani, S. Salinari, F. Tecchio, P. Vitali, O. Zanetti, F. Zappasodi, and P. M. Rossini, "Mapping distributed sources of cortical rhythms in mild Alzheimer's disease. A multicentric EEG study", NeuroImage 22 (2004) 57-67.

[4] Miyagi Y, Morioka T, Fukui K, Kawamura T, Hashiguchi K, Yoshida F, Shono T, Sasaki T., "Spatio-temporal analysis by voltage topography of ictal electroencephalogram on MR surface anatomy scan for the localization of epileptogenic areas.", Minim. Invasive Neurosurg., 2005, 48(2): 97-100.

[5] Ebersole J. S., "Defining epileptic foci: past, present, future.", J Clin Neurophysiol, 1997, 14: 470-483.

[6] Scherg M., "From EEG source localization to source imaging.", Acta Neurol Scand, 1994, 152: 29-30.

[7] Tekgul H., Bourgeois B.F., Gauvreau K., Bergin A.M., "Electroencephalography in neonatal seizures: comparison of a reduced and a full 10/20 montage.", Pediatr Neurol. 2005 Mar; 32(3): $155-61$.

[8] Nayak D, Valentin A, Alarcon G, Garcia Seoane JJ, Brunnhuber F, Juler J, Polkey CE, Binnie CD., "Characteristics of scalp electrical fields associated with deep medial temporal epileptiform discharges.", Clin Neurophysiol. 2004 Jun; 115(6):1423-35.

[9] Skrandies W, Dralle D., "Topography of spectral EEG and late VEP components in patients with benign rolandic epilepsy of childhood.", $J$ Neural Transm. 2004 Feb;111(2):223-30. Epub 2003 Dec 29.

[10] Clemens B., "Ictal electroencephalography in a case of benign centrotemporal epilepsy.", J Child Neurol. 2002 Apr;17(4):297-300.
[11] Tsai ML, Lo HY, Chaou WT., "Clinical and electroencephalographic findings in early and late onset benign childhood epilepsy with occipital paroxysms.", Brain Dev. 2001 Oct;23(6):401-5.

[12] Crone N.E., Boatman D, Gordon B, Hao L., "Induced electrocorticographic gamma activity during auditory perception.", Clin Neurophysiol. 2001 Apr;112(4):565-82.

[13] Iasemidis, L.D.; Deng-Shan Shiau; Chaovalitwongse, W.; Sackellares, J.C.; Pardalos, P.M.; Principe, J.C.; Carney, P.R.; Prasad, A.; Veeramani, B.; Tsakalis, K., "Adaptive epileptic seizure prediction system”, IEEE Transactions On Biomedical Engineering, VOL. 50, NO. 5, MAY 2003, pp: 616- 627

[14] Delorme A., Makeig S., "EEGLAB: An open source toolbox for analysis of single-trial EEG dynamics including independent component analysis.", Journal of Neuroscience Methods. http://sccn.ucsd.edu/eeglab/download/eeglab_jnm03.pdf.

[15] Casdagli, M.C., Iasemidis, L.D., Gilmore, R.L., Roper, S.N., Savit, R.S., Sackellares, J.C., " Characterizing non-linearity in invasive EEG recordings from temporal lobe epilepsy.", Physica D 99, 381-399, 1996.

[16] Casdagli, M.C., Iasemidis, L.D., Savit, R.S., Gilmore, R.L., Roper, S.N., Sackellares, J.C., 1997. "Nonlinearity in invasive EEG recordings from a patient with temporal lobe epilepsy.", Electroencephalogr. Clin. Neurophysiol. 102, 98-105.

[17] Grassberger, P., Procaccia, I., 1983. "Measuring the strangeness of strange attractors.", Physica D 9, 189.

[18] Babloyantz, A., Destexhe, A., 1986. "Low dimensional chaos in an instance of epilepsy.", Proc. Natl. Acad. Sci. U.S.A. 83, 3513-3517.

[19] Abarbanel, H.D.I., Brown, R., Sidorowich, J.J., Tsimring, L.S., 1993., "The analysis of observed chaotic data in physical systems.", Rev. Mod. Phys. 65, 1331.

[20] W. Chaovalitwongse, L.D. Iasemidis, P.M. Pardalos, P.R. Carney, D.S. Shiau, J.C. Sackellares, S.N., 2000. "Performance of a seizure warning algorithm based on the dynamics of intracranial EEG.", Epilepsy Research 64 (2005) 93-113.

[21] Iasemidis, L.D., Sackellares, J.C., Zaveri, H.P.,Williams,W.J., 1990. "Phase space topography and the Lyapunov exponent of the electrocorticograms in partial seizures.", Brain Topogr. 2, 187-201.

[22] Iasemidis, L.D., Sackellares, J.C., 1991. "The evolution with time of the spatial distribution of the largest Lyapunov exponent on the human epileptic cortex.”, In: Duke, D.W., Pritchard, W.S. (Eds.), Measuring Chaos in the Human Brain. World Scientific, Singapore, pp. 49-82.

[23] Iasemidis, L.D., 1991. "On the dynamics of the human brain in temporal lobe epilepsy.", Ph.D. Dissertation, University of Michigan, Ann Arbor. 